\title{
Indomethacin-induced gastric damage in rats and the protective effect of donkey milk
}

Ebru Tastekin ${ }^{1}$, Suleyman Ayvaz ${ }^{2}$, Ufuk Usta ${ }^{1}$, Nurettin Aydogdu ${ }^{3}$, Ekrem Cancilar ${ }^{2}$, Fulya Oz Puyan ${ }^{1}$

${ }^{1}$ Department of Pathology, Medical Faculty, Trakya University, Edirne, Turkey 2Department of Pediatric Surgery, Faculty of Medicine, Trakya University, Edirne, Turkey

${ }^{3}$ Department of Physiology, Faculty of Medicine, Trakya University, Edirne, Turkey

Submitted: 10 July 2015

Accepted: 20 January 2016

Arch Med Sci 2018; 14, 3: 671-678

DOI: https://doi.org/10.5114/aoms.2016.59645

Copyright $\odot 2016$ Termedia \& Banach

\section{Abstract}

Introduction: Indomethacin is an anti-inflammatory drug with clearly known side effects on gastric mucosa. New treatment and side effect prevention methods are being studied. Donkey milk, as a nutritional support, has recently come into the spotlight with its anti-oxidant features, high antibody content and low allergenic properties. In this study, we investigated donkey milk's possible protective effect against acute gastric mucosal damage by indomethacin.

Material and methods: Four groups, each composed of 8 rats, were created. Rats in the first and third groups were fed with standard rat chow, while those in the second and fourth groups were additionally fed with $25 \mathrm{mg} / \mathrm{kg}$ of donkey milk per day via nasogastric gavage. On the $11^{\text {th }}$ day gastric mucosal damage was induced by oral administration of $30 \mathrm{mg} / \mathrm{kg}$ of indomethacin to the rats in groups 3 and 4 . Six $h$ later all rats were sacrificed and their stomachs were removed for macroscopic and microscopic evaluation as well as biochemical examination of glutathione (GSH) and malondialdehyde (MDA) levels. Tumor necrosis factor- $\alpha$ (TNF- $\alpha$ ) expression in the gastric mucosa was evaluated immunohistochemically.

Results: In the donkey milk-indomethacin group, total area of erosion and degree of linear ulceration were significantly lower than in the standard food-indomethacin group $(p<0.05)$. Also, GSH levels were increased and MDA levels were decreased significantly in this group. Tumor necrosis factor- $\alpha$ expression was more prevalent and stronger in the gastritis group, while lower expression was observed in the donkey milk group.

Conclusions: Donkey milk was observed to have significant protective effects against gastric damage induced by indomethacin.

Key words: donkey milk, gastric damage, protective effect, indomethacin.

\section{Introduction}

Nonsteroidal anti-inflammatory drugs (NSAIDs) are widely used all over the world. Indomethacin is one of the most widespread NSAIDs owing to its strong analgesic, antipyretic and anti-inflammatory effects [1]. It is also well known that indomethacin has strong damaging effects on gastric mucosa.

Indomethacin-induced mucosal damage can cause serious mucosal erosions, ulcerative lesions and hemorrhage. COX inhibition hampers mucosal blood flow to the stomach, impairs microcirculation and de-

\author{
Corresponding author: \\ Assist. Prof. Ebru Tastekin \\ Department of Pathology \\ Medical Faculty \\ Trakya University \\ 22030 Edirne, Turkey \\ Phone: \\ +90 2842357642-1532 \\ E-mail: ebrutastekin@ \\ hotmail.com
}


creases mucosal secretion. Decreased secretion hinders defensive functions against acid stimulated by consumed food [2]. Gastric mucosal damage via NSAIDs occurs basically due to a local and/or systematic effect [3]. Oral consumption of NSAIDs leads to release of intercellular adhesion molecule-1 in vascular endothelial cells of gastric mucosa. In consequence of the mechanism, massive amounts of neutrophils stick to vascular endothelial cells due to inflammatory cytokines such as tumor necrosis factor $\alpha$ (TNF- $\alpha$ ) and interleukin 1 (IL-1) [4]. Also reactive oxygen species, released by neutrophils, play a key role in oxidation of biomolecules. As a result of the phenomena, vascular endothelial damage decreases blood flow in gastric mucosa and in the end breaks down hemodynamic microcirculation [5]. Locally, NSAIDs directly affect gastric mucosa and stop oxidative phosphorylation. This leads to decreased electron transfer in the mitochondrial membrane and also causes release of cytochrome C. Cytochrome $\mathrm{C}$ creates oxygen radicals which play a major role in the activation of proteases; thus apoptosis takes place $[6,7]$. Additionally, oxidative stress plays a key role in gastric mucosal damage caused by NSAIDs, and strong antioxidants can suppress oxidative damage related to NSAIDs $[6,8,9]$.

Since the end of the $20^{\text {th }}$ century consumption of donkey milk has been on the rise. Especially in Western Europe, infirm and convalescent patients along with infants are increasingly consuming donkey milk [10]. In Italy, donkeys' milk is mainly used for infant nutrition as a natural substitute milk when breast-feeding is not possible or when there is a need to replace bovine milk in dietotherapy of patients affected by cows' milk protein allergy (CMPA) [11]. When compared to cow milk, there is less fat and more protein in the composition of donkey milk, and it has closer properties to human milk. Donkey milk is easier to digest and more palatable, and also it is richer in nutritional value [12]. Rich in vitamins A and B and poly-unsaturated fatty acids, donkey milk also has low levels of cholesterol [13]. Compared to cow milk, the casein/whey protein ratio is lower in donkey milk, and it is poorer in $\beta$-lacto globulin and richer in $\alpha$-lacto globulin and immunoglobulin (Ig) [14]. Also present is epidermal growth factor, which plays a role in maturation of the infant's intestinal mucosa [15].

Donkey milk consumption is widely encouraged in the presence of immune diseases, cow milk allergy, multiple food intolerances and atherosclerosis [16-18]. Anti-proliferative and antitumor activity of donkey milk was also reported in an experimental study [19]. The effects of donkey milk on immune responses lead to an increase in IL-8 and IL- 6 levels, decrease of TNF- $\alpha$ and
IL-1 production [20]. Recent studies have shown the potential anti-inflammatory effect of donkey milk [21]. Its anti-oxidant capacities are also very high, with high levels of vitamin $E$, vitamin $C$ and polyunsaturated fatty acids such as omega 3 and omega 6 [22].

Donkey milk's general characteristics show its potential as a deterrent against gastric mucosal damage owing to its anti-inflammatory and anti-oxidant properties. In this study, we aim to examine donkey milk's protective characteristics against gastric mucosal damage induced by indomethacin, which is a widely used NSAID.

\section{Material and methods}

This experimental study was carried out in the pathology laboratory of Trakya University Hospital and in the experimental laboratory of animals, Edirne, Turkey between October 2013 and May 2014. Fresh and unprocessed donkey milk was obtained daily from Koruköy Farm (Koruköy Çiftliği, Kırklareli).

\section{Experimental animals}

Male Sprague-Dawley rats (280-320 g) were provided by Trakya University experimental laboratory of animals. The local ethics committee for animal experiments of Trakya University approved the study. All rats were kept in standard laboratory conditions (temperature $22-24^{\circ} \mathrm{C}$, humidity $60 \%$ etc.). Four groups, each with 8 rats, were formed. Rats in the first and third groups were fed with standard rat chow, while those in the second and fourth groups were fed with $25 \mathrm{mg} / \mathrm{kg}$ donkey milk each morning for 10 days, in addition to rat chow. The daily milk consumption was in accordance with the literature data $(200-500 \mathrm{ml} /$ day depending on age) [17].

On the $11^{\text {th }}$ day gastric damage was induced by oral exposure of $30 \mathrm{mg} / \mathrm{kg}$ indomethacin to the rats in the $3^{\text {rd }}$ and $4^{\text {th }}$ groups. All rats were sacrificed $6 \mathrm{~h}$ after indomethacin intake [6]. All stomachs were photographed and mucosal damage was scored macroscopically. Half of the stomach, containing a part of the damaged mucosa, was sent to the physiology laboratory for detecting glutathione (GSH) and malondialdehyde (MDA) levels of the tissues, and they were transported in $-80^{\circ} \mathrm{C}$ freezers during the laboratory steps. The other half was sent to the pathology laboratory in $10 \%$ buffered formaldehyde, for histopathologic immunohistochemical examination.

\section{Antioxidant evaluation}

The stomach tissue sample was mixed with $150 \mathrm{mM} \mathrm{KCl}$ and then homogenized. The homogenized gastric mucosal tissue was centrifuged at $2000 \mathrm{~g}$ for $10 \mathrm{~min}$. The MDA levels of the tissue 
contents, useful as a gauge for lipid peroxidation, were assayed as thiobarbituric acid reacting substances [23]. Two hundred microliters of sample was mixed with $0.2 \mathrm{ml}$ of $8.1 \%$ sodium dodecyl sulfate, $1.5 \mathrm{ml}$ of $20 \%$ acetic acid $(\mathrm{pH}=3.5)$, $0.6 \mathrm{ml}$ of distilled water and $1.5 \mathrm{ml}$ of $0.8 \%$ thiobarbituric acid. The composition was heated to $95^{\circ} \mathrm{C}$ for an hour. After cooling, $5.0 \mathrm{ml}$ of $n$-butanol pyridine (15: 1, v/v) mixed with $1.0 \mathrm{ml}$ distilled water was added to the compound. The compound was centrifuged at $2000 \mathrm{rpm}$ for $10 \mathrm{~min}$ at $25^{\circ} \mathrm{C}$. Absorbance of the organic layer was measured at $532 \mathrm{~nm}$. Malondialdehyde was measured using an extinction coefficient of $1.56 \times 10^{5} \mathrm{M}^{-1} \mathrm{~cm}^{-1}$ and expressed as nanomoles of MDA per gram of wet tissue (nmol/g). For the GSH measurement, $0.5 \mathrm{ml}$ of the supernatant was harvested and treated with $0.3 \mathrm{~mol} / / \mathrm{Na}_{2} \mathrm{HPO}_{4} \cdot 2 \mathrm{H}_{2} \mathrm{O}$ solution at a $2 \mathrm{ml}$ volume. Then, this mixture was treated with $0.2 \mathrm{ml}$ of dithiobisnitrobenzoate $(0.4 \mathrm{mg} / \mathrm{ml}, 1 \%$ sodium citrate). The optical density was measured at a wavelength of $412 \mathrm{~nm}$, and the concentration was calculated accordingly. This was in accordance with the method of Ellman [24]. The composition of GSH was observed with a spectrometer at $412 \mathrm{~nm}$. The results were presented as $\mu \mathrm{mol} / \mathrm{ml}$.

\section{Macroscopic assessment of gastric mucosal damage (scoring of macroscopic gastric mucosal damage)}

All macroscopic evaluations were performed just after scarification of the rats and before sending half of the stomach to the physiology laboratory. A semi-quantitative scale was determined based on severity of hyperemia and hemorrhagic erosions and scored from 0 to 4 as follows: 0 - normal mucosa; 0.5 - hyperemia; 1 - one or two erosions; 2 - severe erosions; 3 - very severe erosions; 4 - mucosal lesions throughout the stomach (hemorrhagic erosions, hyperemia-vascular congestions) [25].

Gastric mucosal erosions were measured under a dissecting microscope with a transparent sheet with $1 \mathrm{~mm}^{2}$ scales on it. The proportion of the damaged area to whole mucosal area was noted. The percentage of indomethacin-induced damage was calculated in comparison to the control group.

\section{Microscopic evaluation of gastric mucosal damage (scoring of gastric mucosal damage histologically)}

Tissue samples were obtained from one half of the stomach and were saved for histologic assessment. Five micrometer sections were obtained from paraffin-embedded tissues and stained with hematoxylin and eosin. Tissue slides were examined under a light microscope (Olympus BX51,
Olympus Co., Shinjuku-ku, Tokyo, Japan). The intensity of gastric mucosal damage was scored from 0 to 3 as follows: 0 - normal; 1 - mucosal erosion; 2 - mucosal and submucosal ulceration; 3 - ulcer extending to muscularis propria $[6,26]$.

Mucosal and submucosal inflammation was also evaluated, and the intensity of the inflammation was scored from 0 to 3 as follows: 0 - none; 1 - mild; 2 - moderate; 3 - severe [6].

\section{Immunohistochemical assessment}

Four-micrometer thick sections which were obtained from formalin-fixed and paraffin-embedded tissue blocks of the gastric mucosal specimens were processed in the pathology laboratory for immunohistochemical examination. Tumor necrosis factor- $\alpha$ antibody was applied to the tissue slides in an automatic immunohistochemical staining device (Ventana BenchMark XT) with a standard incubation procedure for the antibody. Intensity of the immune reaction was scored as follows: 0 - no reaction; 0.5 - weak reaction; 1 - focal strong reaction; 2 - diffuse strong reaction [7].

\section{Statistical analysis}

All data analyses were carried out with the SPSS 20.0 for Windows program package. Macroscopic damage score, gastric mucosal damage, inflammation score, MDA-GSH parameters and immunohistochemical results were evaluated by the Kruskal-Wallis test followed by the Mann-Whitney $U$-test. Differences were considered statistically significant for $p<0.05$.

\section{Results}

Macroscopic and histopathologic evaluation

The control group and group 2 had no mucosal or submucosal damage on macroscopic evaluation. On the other hand, macroscopically observable erosions and ulcers were present in group 3. Ulcers were generally widespread, linear, and the severity of macroscopic mucosal damage was significant in groups 3 and 4 compared to the control group ( $p<0.05$ ). However, macroscopic mucosal damage in group 4 was significantly lower than in group $3(p<0.05)$ (Table I, Figures 1, 2).

Except for sparse and suspicious hyperemia and edema, there was no prominent lesion in groups 1 and 2 on microscopic evaluation. Deep, widespread ulcers were observed in group 3 , while group 4 generally had mucosal erosions and ulcerations limited to superficial mucosa. Group 3 had deep ulcers reaching the submucosa, while group 4 had none $(p<0.05)$ (Table I, Figure 2$)$.

Mucosal and submucosal inflammation was either absent or very scarce in groups 1 and 2. Group 3 
Ebru Tastekin, Suleyman Ayvaz, Ufuk Usta, Nurettin Aydogdu, Ekrem Cancilar, Fulya Oz Puyan

Table I. Measurement of histopathological response in all groups

\begin{tabular}{|c|c|c|c|}
\hline Groups & $\begin{array}{c}\text { Macroscopic mucosal } \\
\text { damage }\end{array}$ & $\begin{array}{l}\text { Histological gastric mucosal } \\
\text { damage }\end{array}$ & Inflammatory score \\
\hline 1 & $0.13 \pm 0.35$ & $0.63 \pm 0.18$ & $0.25 \pm 0.47$ \\
\hline 2 & $0.00 \pm 0.00$ & $0.63 \pm 0.18$ & $0.13 \pm 0.35$ \\
\hline 3 & $2.25 \pm 0.46$ & $2.00 \pm 0.76$ & $2.38 \pm 0.52$ \\
\hline 4 & $1.50 \pm 0.54$ & $0.88 \pm 0.52$ & $1.25 \pm 0.46$ \\
\hline$P$-value & $\begin{array}{l}<0.001^{\beta} \\
0.001^{\alpha}\end{array}$ & $\begin{array}{l}<0.001^{\beta} \\
0.009^{\alpha}\end{array}$ & $\begin{array}{l}<0.001^{\beta} \\
0.011^{\alpha}\end{array}$ \\
\hline
\end{tabular}

Group 1 - control, group 2 - standard rat chow + donkey milk, group 3-standard rat chow + indomethacin, group 4 - standard rat chow + donkey milk + indomethacin; ${ }^{\beta}$ - group 1 and group $3,{ }^{\alpha}$ - group 3 and group 4.

A

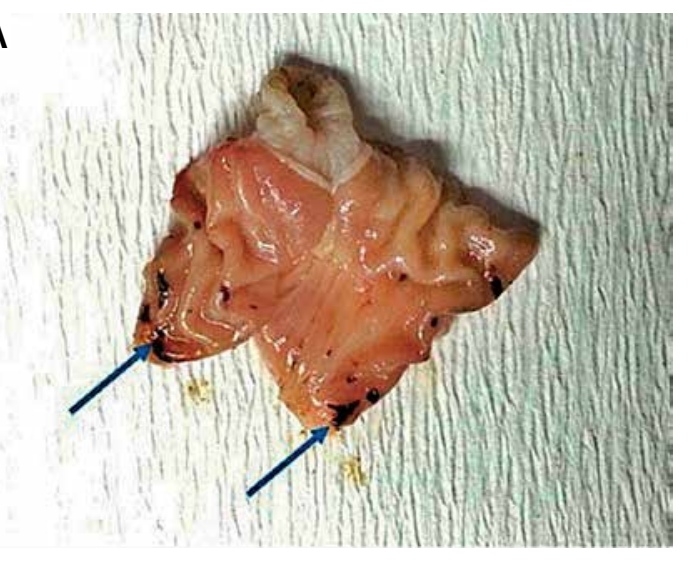

C

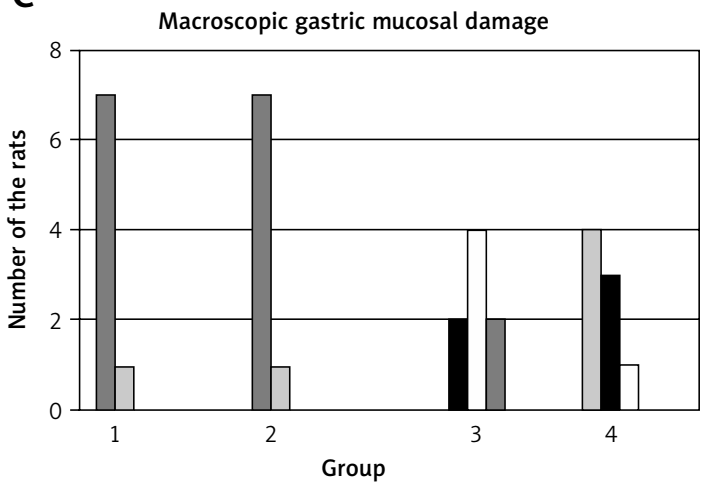

$\square$ Normal

$\square$ One/two lesion

- Very severe lesions $\square$ Hyperemia

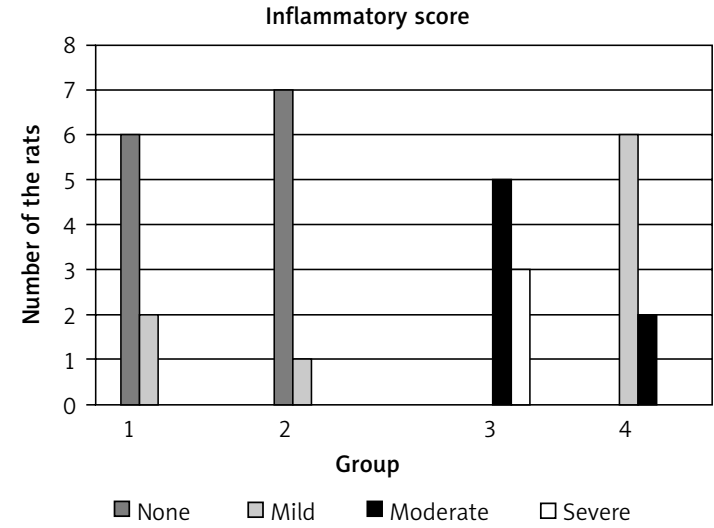

B

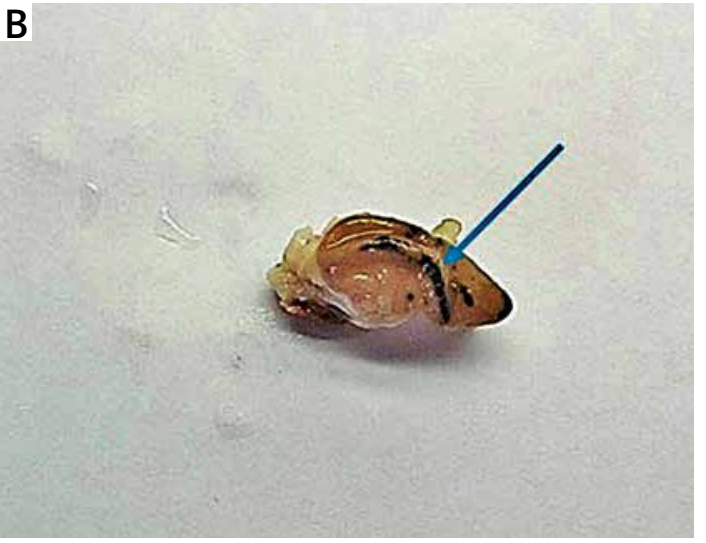

Histologic gastric mucosal damage

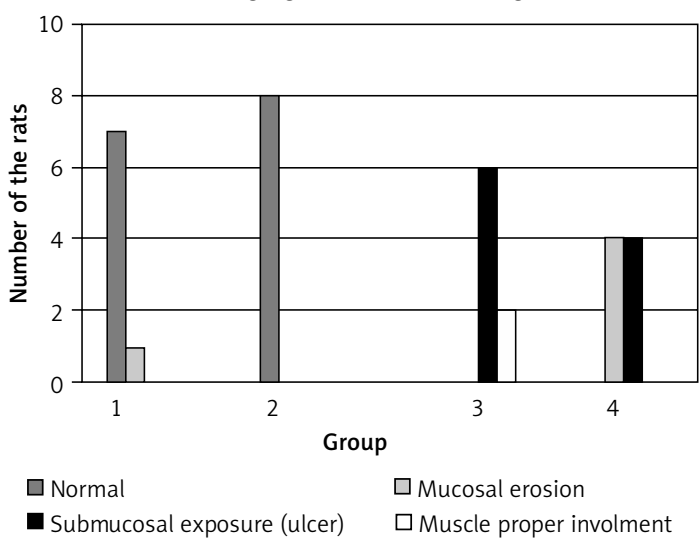

Figure 1. Macroscopic features of rat's stomach. Observable linear hemorrhagic erosions and ulcers (arrows) were present in indomethacin-administered groups. Ulcers were generally widespread, linear, deeply fissured (A - fresh tissue, B - formalin-fixed tissue). Prefeeding with donkey milk was diminished to the grade of macroscopic gastric mucosal injury, histologic gastric mucosal injury and submucosal inflammation caused by indomethacin (C) 
A

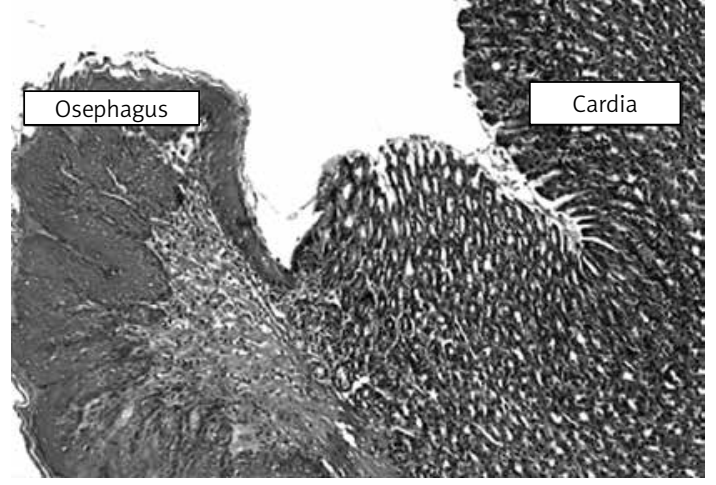

C

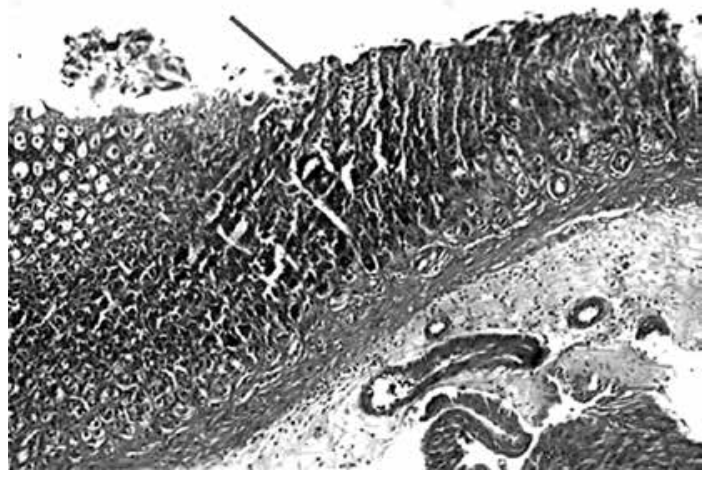

$\mathrm{E}$

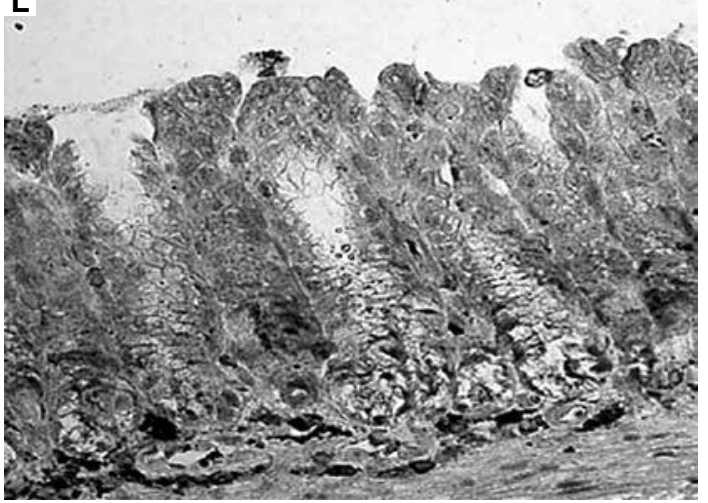

G

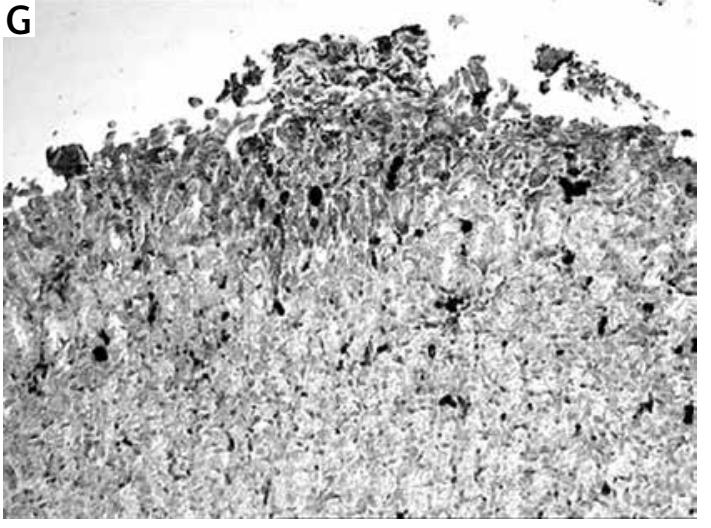

B
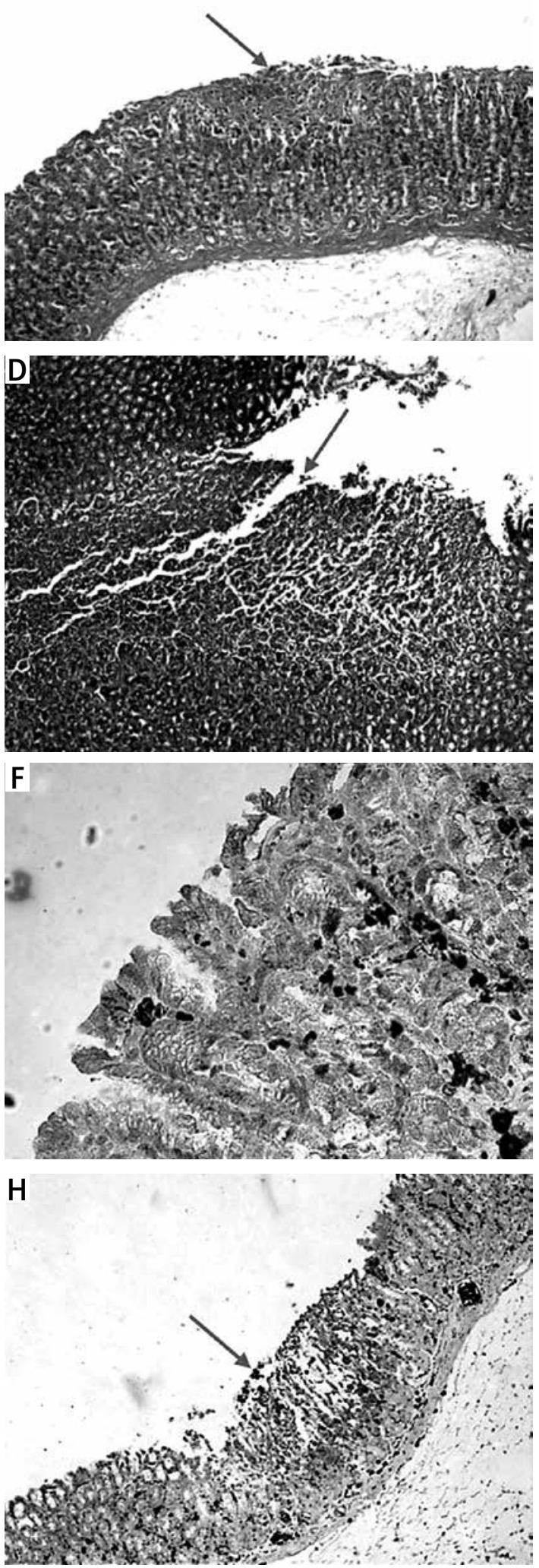

Figure 2. Microscopic features of gastric mucosa in groups. Normal esophagus and cardia mucosa in groups 1 and 2 (A). Mucosal erosion and occasional superficial ulceration (arrow) in group 4 (B). In group 3, deep, widespread ulcers (arrows) (C, D) $(H+E, \times 50)$. There was no/minimal TNF positivity in groups 1 and $2(E, \times 200)$. On the other hand in group 3 strong TNF- $\alpha$ positivity (arrow) was seen especially in ulcerated mucosa and vicinity $(\mathbf{G}, \times 100$, $\mathrm{H}, \times 50)$. In group 4 weaker staining was seen compared to group $3(\mathrm{~F}, \times 200)$ 
had moderate/severe inflammation, whereas group 4 generally had mild/moderate inflammation which was significantly lower than group 3 $(p<0.05)$ (Table I, Figure 2).

\section{Changes in MDA and GSH levels in gastric mucosa}

Malondialdehyde concentrations were found to be $0.12 \pm 0.03 \mu \mathrm{mol} / \mathrm{ml}$ in group 1 and 0.32 $\pm 0.04 \mu \mathrm{mol} / \mathrm{ml}$ in group 3 . These findings indicate that the concentration of MDA in the gastric mucosa was significantly increased in the indomethacin group $(p<0.05)$. Group 2 rats, which were fed with donkey milk in addition to standard rat food, had an MDA rate of $0.14 \pm 0.03 \mu \mathrm{mol} / \mathrm{ml}$. Those rates did not show any significant difference from the control group. Group 4 rats, which were fed similarly to group 2 and which were also given indomethacin, had an MDA level of 0.20 $\pm 0.08 \mu \mathrm{mol} / \mathrm{ml}$. This result was significantly lower than the results of group 3 ( $p<0.05)$ (Table II).

Glutathione levels were investigated in all groups. Glutathione concentration in the control group (group 1) was statistically higher ( $p<$ 0.05 ) than group 3. Glutathione concentration of group 2 showed no significant difference from the control group, whereas GSH concentration of group 4 was significantly higher than the concentration of group $3(p<0.05)$ (Table II).

\section{TNF- $\alpha$ expression}

Immunohistochemically there was no TNF- $\alpha$ expression in groups 1 and 2 except for very weak positivity in a few rats. However, in the groups which had indomethacin-induced gastritis, there was strong positivity for TNF- $\alpha$ and there was a statistically significant difference compared to groups 1 and $2(p<0.05)$. Especially ulcerated mucosa and adjacent inflamed mucosa showed stronger expression. Group 4 showed weaker expression for TNF- $\alpha$ (Table II).

Table II. Malondialdehyde, glutathione and tumor necrosis factor levels in all groups

\begin{tabular}{|lccc|}
\hline Groups & MDA & GSH & TNF \\
\hline 1 & $0.12 \pm 0.03$ & $1.56 \pm 0.14$ & $0.20 \pm 0.45$ \\
\hline 2 & $0.14 \pm 0.03$ & $1.61 \pm 0.09$ & $0.13 \pm 0.35$ \\
\hline 3 & $0.32 \pm 0.04$ & $0.95 \pm 0.10$ & $1.75 \pm 0.46$ \\
\hline 4 & $0.20 \pm 0.08$ & $1.46 \pm 0.21$ & $0.88 \pm 0.64$ \\
\hline & $0.003^{\beta}$ & $0.003^{\beta}$ & $0.003^{\beta}$ \\
& $0.009^{\alpha}$ & $0.001^{\alpha}$ & $0.011^{\alpha}$ \\
\hline
\end{tabular}

MDA - malondialdehyde, GSH - glutathione, TNF - tumor necrosis factor. Group 1 - control, group 2 - standard rat chow + donkey milk, group 3 - standard rat chow + indomethacin, group $4-$ standard rat chow + donkey milk + indomethacin; ${ }^{\beta}-$ group 1 and group $3,{ }^{\alpha}-$ group 3 and group 4.

\section{Discussion}

The NSAIDs are still widely used all over the world for their analgesic and anti-inflammatory properties [26, 27]. Indomethacin has widespread application in this category. However, it is well known that indomethacin can cause diffuse and severe erosions and ulcers in the gastric mucosa, and these side effects seriously restrict indomethacin usage $[28,29]$. Generation of free oxygen radicals and lipid peroxide especially plays a key role in the progression of the gastric mucosal lesions caused by indomethacin [3, 30, 31]. Immunohis tochemical and biochemical data of the present study clearly show that donkey milk has anti-inflammatory and anti-oxidant effects on gastric mucosal damage induced by indomethacin.

Oxidative damage becomes more pronounced with lipid peroxidation, which is important in physiopathology of gastric damage, concerning damage to the cell and the cell membrane. In previous studies the amount of lipid peroxidation and superoxide dismutase (SOD) was shown to be related to gastric mucosal damage induced by indomethacin as well as enzyme activity such as catalase and glutathione peroxidase [32, 33]. Thus, consumption of substances that can increase the activity of these enzymes for protection of the gastric mucosa from the effects of indomethacin is a potential solution in the first step. In the present study histopathological evaluation showed that, of the two groups which were exposed to indomethacin, group 3 had more numerous and deeper ulcers when compared to group 4 rats, which were fed with donkey milk and which had smaller and more superficial ulcers. This important result provides further evidence for the protective effect of donkey milk on gastric mucosa.

There are many studies documenting various substances with protective and therapeutic properties against indomethacin-induced gastric ulcer [32-34]. In particular, the antioxidant effect of selenium is well known, and its curative effect on gastric mucosal oxidative stress is widely documented [34]. Furthermore, the anti-oxidant properties and curative effect of L-carnitine on gastric mucosal damage have been subject to various studies [35] Kim et al. [6] investigated the effect of grape seeds on indomethacin-induced gastritis, and they observed the rise in GSH levels with protective-curative properties. Furthermore, Kim et al. demonstrated selenium's curative effect which leads to high levels of GSH and low levels of MDA [1]. Owing to our significant observations in group 4, high GSH levels as an indicator of anti-oxidant activity were also investigated in our research. There are also several ex-vivo, in-vivo and in-vitro studies concerning donkey milk's anti-oxidant effects, but not on gastric mucosa $[17,22]$. Our findings indicate 
that a low level of GSH is observable in indomethacin-induced gastric damage and the donkey milk fed group had similar levels of GSH to the control group. Furthermore, MDA levels were high in group 3, while group 4 had lower levels of MDA, a finding closer to the control group. On the basis of these findings it can be suggested that donkey milk has a high antioxidant capacity and it can protect the gastric mucosa against indomethacin.

Recent studies claim that donkey milk can also possess anti-inflammatory and anti-microbial properties [14, 21]. Various papers have reported high levels of lysozyme, lactoferrin, $\alpha$-lactoglobulin and immunoglobulin contents of donkey milk [21, 34, 35]. Such attributes function against possible infections and other possible causes of inflammation in the digestive system [36-38]. In our study, group 4, which was additionally fed with donkey milk, had significantly lower levels of inflammation compared to group 3. This finding also supports the anti-inflammatory property of donkey milk.

An NSAID-associated increase in the concentration of local inflammatory mediators (pro-inflammatory cytokines), such as IL-1, TNF- $\alpha$, IL-8, IL-17 and IL-22, does not lead to an increase in concentration of systemic cytokines in blood serum. Tumor necrosis factor $\alpha$ (or cachectin) is an adipokine involved in systemic inflammation and is a member of the cytokine family that stimulates the acute phase reaction. It is produced mainly by activated macrophages, although it can be produced by CD4+ lymphocytes, NK cells, neutrophils, mast cells, eosinophils, and neurons. Accordingly, to make a diagnosis of NSAID-induced damage of the gastric mucosa using blood sampling without esophagogastroduodenoscopy for chronic NSAIDs users, a substantial effort should be made to identify a novel type of marker indicating inflammation of the gastric mucosa $[37,39]$. There are very few studies involving donkey milk's effect on serum TNF- $\alpha$ level, and still there is no such study with donkey milk regarding the local concentration of TNF- $\alpha$ in gastric mucosal tissue in the current literature. Donkey milk shows direct anti-oxidant features via high concentration of polyunsaturated fatty acids (especially omega 3 and omega 6) and also high concentrations of vitamins $C$ and $E$. On the other hand, donkey milk shows indirect effects against inflammation with its antimicrobial effects via lysozyme and lactoferrin proteins. Also, the anti-oxidant effect of donkey milk may lead to an indirect anti-inflammatory effect. Studies evaluating the anti-inflammatory effect by ELISA should be planned in the future to support this result.

Future studies about COX-2, IL-1, IL-6, IL-8 and IL-17 and other important mediators should be carried out regarding the possible effect of donkey milk on pro-inflammatory cytokines. Our study showed a decrease of indomethacin-induced gastric muco- sal damage by initial consumption of donkey milk. There are few studies about donkey milk's effects on bacterial and viral agent based inflammations [10], and none of them are conclusive.

Lactoferrin and lysozyme content of donkey milk confers antimicrobial properties on the milk. Previous studies have shown that donkey milk consumption can inhibit Escherichia coli and Listeria monocytogenes infections in gastric mucosa. Antimicrobial activity can be used to limit gastric mucosal damage caused by infective agents [10]. We hold the opinion that future studies should be conducted on donkey milk's possible effect against Helicobacter pylori infection, which is a widespread problem leading to acute gastritis and gastric ulcers.

Donkey milk is also rich in vitamins and minerals, and it has low fat levels and a pH level very close to human milk; therefore it is beneficial to patients who are in need of nutritional support.

In conclusion, our findings indicate that donkey milk with its anti-inflammatory and anti-oxidant properties can protect the gastric mucosa from the gastric damage induced by indomethacin. The beneficial findings obtained from this study can lead to further research regarding microbiological assessment, and more elaborate research may reveal even more benefits of donkey milk. Also studies evaluating the healing effect of donkey milk on gastric damage and inflammation ought to be conducted.

\section{Conflicts of interest}

The authors declare no conflict of interest.

\section{References}

1. Kim JH, Kim BW, Kwon HJ, Nam SW. Curative effect of selenium against indomethacin-induced gastric ulcers in rats. J Microbiol Biotechnol 2011; 21: 400-4.

2. Zaki SM, Mohamed EA. Effect of glucocorticoids on indomethacin-induced gastric ulcer in the adult male albino rat - histological, morphometric aid electron microscopy study. Arch Med Sci 2014; 10: 381-8.

3. Langenbach R, Morham SG, Tiano HF, et al. Prostaglandin synthase 1 gene disruption in mice reduces arachidonic acid-induced inflammation and indomethacininduced gastric ulceration. Cell 1995; 83: 483-92.

4. Santucci L, Fiorucci S, Giansanti M, Brunori PM, Di Matteo FM, Morelli A. Pentoxifylline prevents indomethacin induced acute gastric mucosal damage in rats: role of tumour necrosis factor alpha. Gut 1994; 35: 909-15.

5. Yoshikawa T, Naito Y. The role of neutrophils and inflammation in gastric mucosal injury. Free Radic Res 2000; 33: 785-94.

6. Kim TH, Jeon EJ, Cheung DY, et al. Gastroprotective effects of grape seed proanthocyanidin extracts against nonsteroid anti-inflammatory drug-induced gastric injury in rats. Gut Liver 2013; 7: 282-9.

7. Murphy MP, Smith RA. Drug delivery to mitochondria: the key to mitochondrial medicine. Adv Drug Deliv Rev 2000; 41: 235-50. 
8. Pohle T, Brzozowski T, Becker JC, et al. Role of reactive oxygen metabolites in aspirin-induced gastric damage in humans: gastroprotection by vitamin C. Aliment Pharmacol Ther 2001; 15: 677-87.

9. Zamora Z, Gonzalez R, Guanche D, et al. Ozonized sunflower oil reduces oxidative damage induced by indomethacin in rat gastric mucosa. Inflamm Res 2008; 57: 39-43.

10. Tidona F, Sekse C, Criscione A, et al. Antimicrobial effect of donkeys' milk digested in vitro with human gastrointestinal enzymes. Int Dairy J 2011; 21: 158-65.

11. Muraro MA, Giampietro PG, Galli E. Soy formulas and nonbovine milk. Ann Allergy Asthma Immunol 2002; 89: 97-101.

12. Uniacke-Lowe T, Huppertz T, Fox PF. Equine milk proteins: chemistry, structure and nutritional significance. Int Dairy J 2010; 20: 609-29.

13. Solaroli G, Pagliarini E, C. P. Composition and nutritional quality of mare's milk. Ital J Food Sci 1993; 5: 3-10.

14. Salimei E, Fantuz F, Coppola R, Chiofalo B, Polidori P, Varisco G. Composition and characteristics of ass's milk. Anim Res 2004; 53: 67-78.

15. Scafizzari M, Giannico F, Potere O, et al. Epidermal growth factor (EGF) in mare and ass milk: a preliminary investigation. Ital J Anim Sci 2009; 8: 737.

16. Carroccio A, Cavataio F, Montalto G, D’Amico D, Alabrese L, Iacono G. Intolerance to hydrolysed cow's milk proteins in infants: clinical characteristics and dietary treatment. Clin Exp Allergy 2000; 30: 1597-603.

17. Monti G, Bertino E, Muratore MC, et al. Efficacy of donkey's milk in treating highly problematic cow's milk allergic children: an in vivo and in vitro study. Pediatr Allergy Immunol 2007; 18: 258-64.

18. Tafaro A, Magrone T, Jirillo F, et al. Immunological properties of donkey's milk: its potential use in the prevention of atherosclerosis. Curr Pharm Des 2007; 13: 3711-7.

19. Mao XY, Gu JN, Sun Y, et al. Anti-proliferative and antitumour effect of active components in donkey milk on A549 human lung cancer cells. Int Dairy J 2009; 19: 703-8.

20. Amati L, Marzulli G, Martulli M, et al. Donkey and goat milk intake and modulation of the human aged immune response. Curr Pharm Des 2010; 16: 864-9.

21. Jirillo F, Jirillo E, Magrone T. Donkey's and goat's milk consumption and benefits to human health with special reference to the inflammatory status. Curr Pharm Design 2010; 16: 859-63.

22. Simos Y, Metsios A, Verginadis I, et al. Antioxidant and anti-platelet properties of milk from goat, donkey and cow: an in vitro, ex vivo and in vivo study. Int Dairy J 2011; 21: 901-6.

23. Ohkawa H, Ohishi N, Yagi K. Assay for lipid peroxides in animal tissues by thiobarbituric acid reaction. Anal Biochem 1979; 95: 351-8.

24. Ellman GL. Tissue sulfhydryl groups. Arch Biochem Biophys 1959; 82: 70-7.

25. Dokmeci D, Akpolat M, Aydogdu N, Doganay L, Turan FN. L-carnitine inhibits ethanol-induced gastric mucosal injury in rats. Pharmacol Rep 2005; 57: 481-8.

26. Cashin $\mathrm{CH}$, Dawson W, Kitchen EA. The pharmacology of benoxaprofen (2-[4-chlorophenyl]-alpha-methyl-5-benzoxazole acetic acid), LRCL 3794, a new compound with antiinflammatory activity apparently unrelated to inhibition of prostaglandin synthesis. J Pharm Pharmacol 1977; 29: 330-6.

27. Bjarnason I, Hayllar J, MacPherson AJ, Russell AS. Side effects of nonsteroidal anti-inflammatory drugs on the small and large intestine in humans. Gastroenterology 1993; 104: 1832-47.

28. Beck WS, Schneider HT, Dietzel K, Nuernberg B, Brune K. Gastrointestinal ulcerations induced by anti-inflammatory drugs in rats. Physicochemical and biochemical factors involved. Arch Toxicol 1990; 64: 210-7.

29. Tenenbaum J. The epidemiology of nonsteroidal anti-inflammatory drugs. Can J Gastroenterol 1999; 13: 119-22.

30. Lanza FL. Endoscopic studies of gastric and duodenal injury after the use of ibuprofen, aspirin, and other nonsteroidal anti-inflammatory agents. Am J Med 1984; 77 19-24.

31. Vaananen PM, Meddings JB, Wallace JL. Role of oxygen-derived free-radicals in indomethacin-induced gastric injury. Am J Physiol 1991; 261: G470-5.

32. Delsoldato P, Foschi D, Benoni G, Scarpignato C. Oxygen free-radicals interact with indomethacin to cause gastrointestinal injury. Agents Actions 1986; 17: 484-8.

33. Iwasaki Y, Matsui T, Arakawa Y. The protective and hormonal effects of proanthocyanidin against gastric mucosal injury in Wistar rats. J Gastroenterol 2004; 39: 831-7.

34. Ellinger S, Linscheid KP, Jahnecke S, Goerlich R, Enbergs $\mathrm{H}$. The effect of mare's milk consumption on functional elements of phagocytosis of human neutrophil granulocytes from healthy volunteers. Food Agric Immunol 2002; 14: 191-200.

35. Derin N, Agac A, Bayram Z, Asar M, Izgut-Uysal VN. Effects of L-carnitine on neutrophil-mediated ischemia-reperfusion injury in rat stomach. Cell Biochem Funct 2006; 24: 437-42.

36. Chiavari C, Coloretti F, Nanni M, Sorrentino E, Grazia L. Use of donkey's milk for a fermented beverage with lactobacilli. Lait 2005; 85: 481-90.

37. Terra X, Montagut G, Bustos M, et al. Grape-seed procyanidins prevent low-grade inflammation by modulating cytokine expression in rats fed a high-fat diet. J Nutr Biochem 2009; 20: 210-8.

38. Vincenzetti S, Polidori P, Mariani P, Cammertoni N, Fantuz F, Vita A. Donkey's milk protein fractions characterization. Food Chem 2008; 106: 640-9.

39. El Eter E, Al Tuwaijiri A, Hagar H, Arafa M. In vivo and in vitro antioxidant activity of ghrelin: attenuation of gastric ischemic injury in the rat. J Gastroen Hepatol 2007; 22: 1791-9. 